\title{
ANALYZING THE IMPACT OF KEY PARAMETERS OF VEHICLE MANAGEMENT POLICIES IN A UNIFIED AMHS
}

\author{
Ahmed Ben Chaabane ${ }^{1,2}$ \\ Stéphane Dauzère-Pérès ${ }^{1}$ \\ Claude Yugma ${ }^{1}$ \\ ${ }^{1}$ Department of Manufacturing Sciences and Logistics \\ Ecole des Mines de Saint-Etienne, CMP Georges Charpak \\ 880 route de Mimet \\ F-13541 Gardanne, FRANCE
}

\author{
Lionel Rullière ${ }^{2}$ \\ Gilles Lamiable ${ }^{2}$ \\ ${ }^{2}$ Department of Industrial Engineering \\ STMicroelectronics \\ 850 rue Jean Monnet \\ F-38926 Crolles, FRANCE
}

\begin{abstract}
This paper deals with the management of vehicles in the Automated Material Handling System of a semiconductor wafer fabrication facility. Vehicle management policies (allocation of vehicles to transport requests, positioning of idle vehicles,...) strongly impact critical performance indicators, among them the allocation time (delivery time) and the lot cycle time. In the literature, most studies on the impact of vehicle policies on delivery times focused on segregated layouts and generally do not consider minimum service transport policies. We investigate a minimum service policy which consists in keeping a minimum number of available vehicles in bays to quickly answer transport requests. After describing the transport policy, we investigate how to define its key parameters. Using a detailed discrete event simulation model of a real semiconductor manufacturing facility, various simulation studies are performed to analyze the impact of these parameters.
\end{abstract}

\section{INTRODUCTION}

In a semiconductor wafer fabrication facility (fab), hundreds of machines are available in a large area. Moreover, wafers require several hundreds of steps to be completed, and are coming back many times (up to 40) to the same work area. Hence, wafers travel a long distance during their manufacturing process. Moving wafers between machines is thus very frequent and has been automated when the wafer diameter moved from $200 \mathrm{~mm}$ to $300 \mathrm{~mm}$. Wafers are grouped to be produced and transported in lots of at most 25 wafers. Multiple reasons motivated the idea of automated transportation to replace human operators.

- Physical constraints: Carrying lots that are relatively heavy, is tricky. It is a delicate task that affects the physical capacity of operators and may lead to accidents (damaging wafers during a fall).

- Economic constraints: For a 300mm diameter, a wafer costs between 3,000 and 4,500 dollars. This high cost requires to have a reliable system that ensures priority, integrity and wafer protection. Adding to relatively expensive labor costs, semiconductor companies struggle to make products that can compete in terms of price. Thus, the need to automate the production was a necessity in order to reduce payroll and ensure competitiveness.

In addition, a semiconductor manufacturing facility is very expensive (average cost of 3 billion dollars for a $300 \mathrm{~mm}$ fab). This is why it is a priority to optimize the return on investment. It is also important that the automated transportation system guarantees reliable and repeatable service. Repeatability means the capacity of the vehicles to perform the same task in the same conditions. Reliability means the ability of the transport system to guarantee a given delivery time. 
A lot of wafers undergoes three types of actions: Processing, transportation and storage. Hence, the AMHS is required to move, store and help in the processing of a lot. The performance of the fab is linked to the architecture of the AMHS (number of vehicles, ...), the layout of the fab and the rules implemented in the transport management system. As already written, when managing a fleet of transport vehicles in a manufacturing facility, the goal is to guarantee reliable and fast transport times. To reach these objectives, we focus on the management of vehicles (vehicle policies). More precisely, based on a full-scale fab simulation model, we study the impact on the delivery time of key parameters of a "minimum service" transport policy. The objective of a "minimum service" policy is to ensure that, in each area, there is at least a specified number of vehicles and there is at most another specified number of vehicles to avoid idle vehicles and balance vehicles between areas.

The remainder of this paper is organized as follows. Section 2 gives a brief overview of the literature on AMHS and vehicle management. Section 3 describes the features of the "minimum service" transport policy. Sections 4 and 5 focus on the study of the impact of key parameters of this transport policy on the delivery time. Section 6 concludes the paper.

\section{LITERATURE REVIEW}

In the literature, most studies on the impact of vehicle policies on delivery times focused on segregated layouts and generally do not consider minimum service transport policies. In fabs with segregated layouts, moving lots from a source to a destination requires three independent transport commands: One to leave the source area, one to travel along the central loop and, finally, one to move in the destination bay. Unified AMHS layouts are relatively recent, and less studied in the literature. Because vehicles are allowed to move everywhere in the fab, defining appropriate vehicle policies, such as the minimum service policy is important since, for example, all vehicles could be in the same area and not be able to quickly answer transport requests, see (Kiba et al. 2009).

Most authors in the literature consider "classical" transport policies where vehicles are dispatched through the whole facility using dispatching rules to answer transport requests (Nazzal and McGinnis 2005). The drawback of these policies is that they cannot guarantee a minimal assignment time by allocating available vehicles ahead of time, because predicting the transport requests of each area is complicated. As semiconductor manufacturing processes are complex, planning the vehicle to select for a given transport request is difficult. The idea of minimum service is to try to ensure that, at all times, there are enough available vehicles in each area to quickly answer a transport request and to reduce vehicle assignment times, and thus lot delivery times. (Nazzal and McGinnis 2005), (Nazzal and McGinnis 2006), (Kahraman, Gosavi, and Oty 2008) and (Nazzal and McGinnis 2007) try to estimate allocation times using Markov chains. Conventional analytical models are considered to be unable to integrate the vehicle traffic density. (Hammel, Schmidt, and Schops 2012) provide a static model of a transport system using graph theory to track the system weakness and improve global performance indicators. The advantage of this method is the rapidity to get results from aggregated data, but the main drawback is the total absence of dynamics such as traffic jam and vehicle positions.

The research that addressed the management of vehicles is primarily based on mathematical models and simulation approaches with empirical estimates of the input data. At this stage, these studies are not applicable on an industrial case. (Wertz et al. 2008) and (Kiba et al. 2009) briefly explain the policy of balancing vehicles. This policy, based on the definition of a minimum and maximum number of available vehicles by area, manages the available transport resources. To ensure this, various criteria are mentioned such as the need for parking spaces to avoid cluttering the area. The advanced study by (Wertz et al. 2008) presents simulation results without specifying how to define the parameters of the vehicle policy (the number of vehicles by area, number of parking spaces, ...). (Kiba et al. 2009) study this policy through a partial modeling which guarantees the dynamic aspects of the transport system. The major drawback of this work is that the minimum service policy is partially modeled since vehicles are not allocated per area but for the whole fab, i.e. a vehicle can answer a transport request anywhere in the fab. This is different 
from the actual minimum service policy where vehicles can only serve the area in which they are located. In addition, a list of parking spaces is used where vehicles can go to be available to serve the corresponding area. This scenario is opportunistic and does not conform to the actual policy.

We want to show that optimizing the key parameters of the minimum service policy is very important to reduce the allocation time of vehicles. Establishing the relation between the allocation time and the number of transport requests is difficult. This is why we use discrete event simulation to model the dynamics of the system. In this paper, we model in details the minimum service policy in a detailed simulation model of a real 300mm semiconductor manufacturing facility that includes production, transportation and storage of lots.

\section{THE MINIMUM SERVICE POLICY}

The minimum service policy aims at logically managing transport resources to satisfy transport requests in each area of a unified fab.

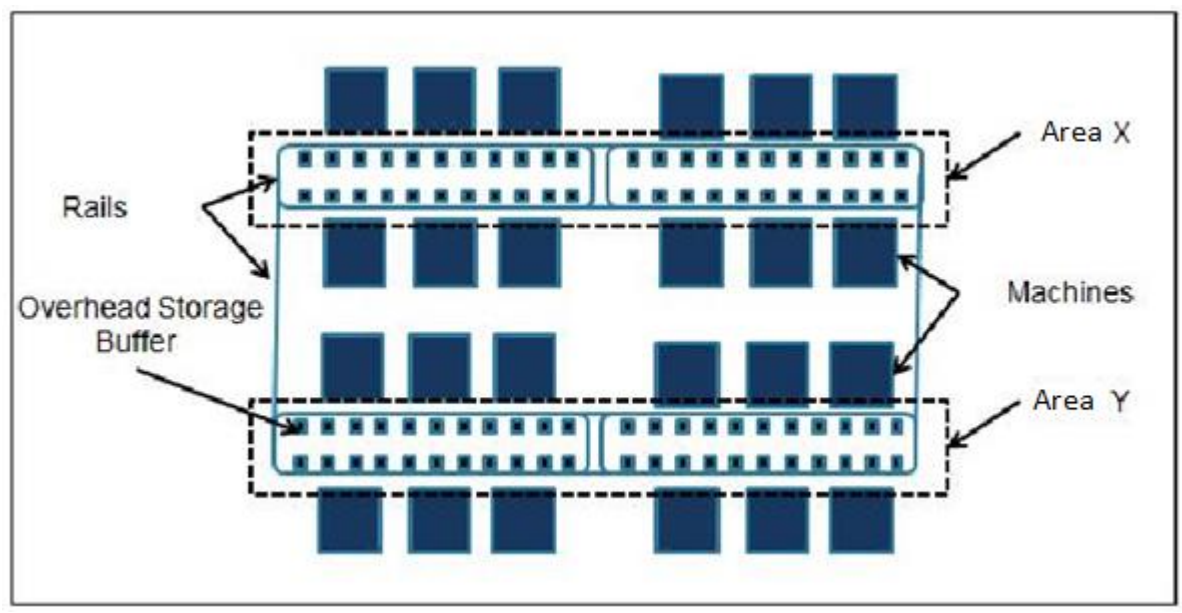

Figure 1: Examples of two areas in a semiconductor manufacturing facility.

Two areas $X$ and $Y$ are considered in Figure 1. Available vehicles in each area are managed using two key parameters:

- LWM (Low Water Mark): Minimum number of available vehicles that should be available in the area.

- HWM (High Water Mark): Maximum number of vehicle that should be available in the area.

The policy works as follows. When a transport demand is triggered in area $X$, the first step is to find an available vehicle in the same area to minimize the allocation time. Otherwise, the demand will wait in a queue until one vehicle in area $X$ becomes free or a free vehicle comes from area $Y$. The Low Water Mark allows to keep available vehicles in the area to satisfy future demands. When the number of available vehicles in the area is strictly smaller than LWM, the system tries to invite empty vehicles from other areas to reach the LWM. On the contrary, when the number of vehicle in the area is strictly larger than the High Water Mark, the system tries to send the additional vehicles to other areas. This policy aims at reducing the delivery times of lots and at balancing transport resources in the fab. To reach this objective, it is necessary to define the right values of LWM and HWM per area and to decide where to look for empty vehicles and to where extra vehicles should be sent. Figure 2 shows that the delivery time is composed of numerous steps which starts by looking for an available transport resource (allocation time) and ends by unloading the lot at the destination. Going to source time, loading time, transport time and unloading time 
are parameters that are usually well established. However, because of its variability, the allocation time is the parameter that impacts the most the delivery time.

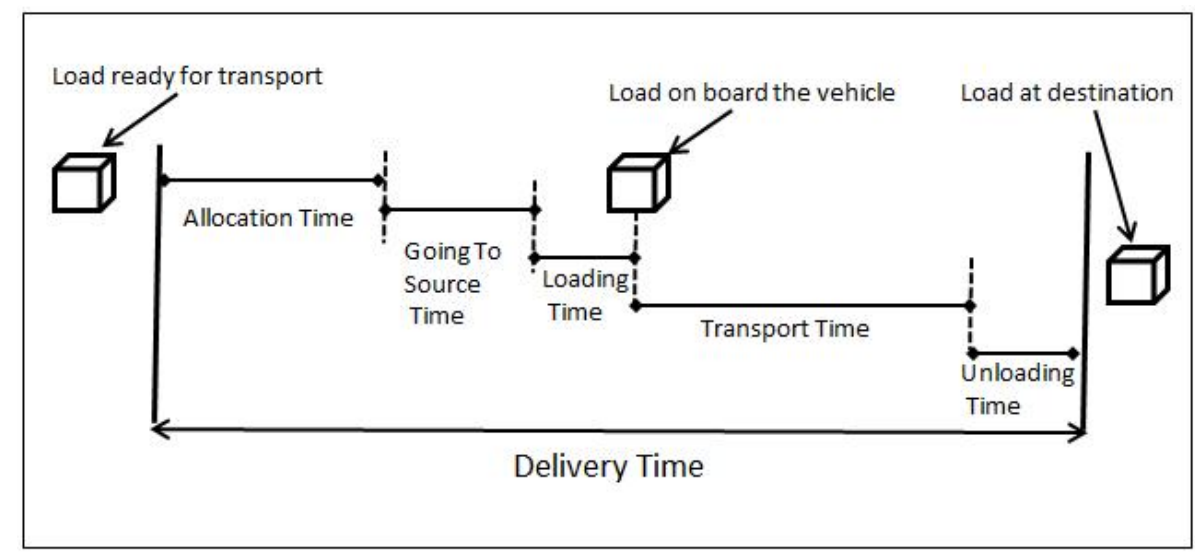

Figure 2: Allocation time as a main component of delivery time.

To estimate the delivery time, two scenarios must be considered. In the first scenario, an available vehicle is in the area and immediately reserved for the transport request and the allocation time is null. In the second scenario, there is no available vehicle in the area and the lots waits for the balancing system to send an available vehicle to the area. The resulting allocation time depends on:

- Where is the idle vehicle looked for (distance between areas)?

- Blocking events in the route to the area (traffic jam or stopped vehicle)?

- The impact in the area where the vehicle is taken (the number of transport requests in each area)?

The allocation time is function of the distance between the source area and the destination area, the probability of blocking events during the trip and the number of transport requests in the destination area. It is very difficult to define this function, but we can study the impact of varying the parameters on allocation times using discrete event simulation. To do this, we modeled the real $300 \mathrm{~mm}$ fab of STMicroelectronics in Crolles. We want to study the impact of key parameters (LWM and HWM) of the minimum service policy on the allocation times of vehicles. The right definition of HWM and LWM allows vehicles to travel shorter distances to satisfy transport requests and potentially avoid transport requests.

\section{STUDY OF THE LOW WATER MARK}

In this section, we analyze the interaction between the values of LWM and the number of transport requests in each area. It is important to differentiate between the transport requests that remain in the area, and thus for which the vehicles stay in the area, and the transport requests that leave the area, for which the vehicles are lost for the area.

\subsection{INTERACTIONS BETWEEN LWM AND THE DEGREE OF FREEDOM OF THE AMHS}

Normally, each area requires an available vehicle to satisfy a transport request. Through simulation, we study the impact of the flow variation of each area on its LWM. Typically, an area with a large number of transport requests would need more vehicles than an area with less transport requests. Let us denote by NbTrans $j$ the daily number of transport requests in area $j$, and by $N T$ the total number of vehicles. Also, let us define $L$ as the "degree of freedom". This parameter helps to specify how many vehicles are available to ensure balancing between areas. More precisely, $L=20 \%$ means that $80 \%$ of the vehicles are 
used to satisfy the LWMs. We propose to define the $L W M$ of area $j$ as:

$$
L W M_{j}=\frac{\text { NbTrans }_{j}}{\sum_{i=1}^{n}\left(\text { NbTrans }_{i}\right)} \times N T \times(1-L) .
$$

Table 1 details the characteristics of the four scenarios that were simulated with different values of the degree of freedom $L$.

Table 1: Four scenarios with different LWMs

\begin{tabular}{|c|c|c|c|c|c|}
\cline { 3 - 6 } \multicolumn{2}{c|}{} & \multicolumn{5}{c|}{ LWM } \\
\hline Area & $\begin{array}{c}\text { Nb of transport } \\
\text { requests }\end{array}$ & $\begin{array}{c}\text { Scenario 1 } \\
(L=-100 \%)\end{array}$ & $\begin{array}{c}\text { Scenario 2 } \\
(L=0 \%)\end{array}$ & $\begin{array}{c}\text { Scenario 3 } \\
(L=20 \%)\end{array}$ & $\begin{array}{c}\text { Scenario 4 } \\
(L=40 \%)\end{array}$ \\
\hline 1 & 702 & 3 & 2 & 1 & 1 \\
\hline $\mathbf{2}$ & $\mathbf{1 3 4 5}$ & $\mathbf{6}$ & $\mathbf{3}$ & $\mathbf{2}$ & $\mathbf{2}$ \\
\hline 3 & 434 & 2 & 1 & 1 & 1 \\
\hline 4 & 957 & 4 & 2 & 2 & 1 \\
\hline $\mathbf{5}$ & $\mathbf{1 9 0 3}$ & $\mathbf{8}$ & $\mathbf{4}$ & $\mathbf{3}$ & $\mathbf{2}$ \\
\hline $\mathbf{6}$ & $\mathbf{1 7 4 9}$ & $\mathbf{7}$ & $\mathbf{4}$ & $\mathbf{3}$ & $\mathbf{2}$ \\
\hline 7 & 1553 & 6 & 3 & 3 & 2 \\
\hline 8 & 430 & 2 & 1 & 1 & 1 \\
\hline $\mathbf{9}$ & $\mathbf{2 6 0 5}$ & $\mathbf{1 1}$ & $\mathbf{6}$ & $\mathbf{5}$ & $\mathbf{3}$ \\
\hline 10 & 401 & 2 & 1 & 1 & 1 \\
\hline
\end{tabular}

- In the first scenario, $L=-100 \%$, i.e. the total number of vehicles is not sufficient to satisfy the balancing system for all the areas $\left(\sum_{i=1}^{n} L W M=2 \times N T\right)$.

- In the second scenario, $L=0 \%$, i.e. a total allocation of the transport resources $\left(\sum_{i=1}^{n} L W M=N T\right)$. All the vehicles in the system are balanced between areas.

- In the third scenario, $L=20 \%$, i.e. $\sum_{i=1}^{n} L W M=0.8 \times N T$.

- In the fourth scenario, $L=40 \%$, i.e. $\sum_{i=1}^{n} L W M=0.6 \times N T$.

The ten areas studied in this paper includes four specific areas: Areas 2, 5 and 9 characterized by high throughputs and Area 6 where machines operate by batches of six lots. Given their characteristics, these areas will be more sensitive to the variation of their LWM. The number of transport requests considered in the scenarios matches the number of lots shipped during one day in each area. The duration of the simulation is three days. In the section below, we analyze the impact of varying the degree of freedom $(L)$ on delivery times.

\subsection{ANALYZING THE IMPACT OF THE DEGREE OF FREEDOM}

In the first scenario, the sum of the LWMs is larger than the number of vehicles available in the fab $\left(\sum_{i=1}^{n} L W M=2 \times N T\right)$. This setting has a major drawback since the system is unstable for all areas. The mean and standard deviation of the delivery time for Areas 1, 3, 4, 7, 8 and 10 are relatively large compared to the other scenarios $(\min =389$ seconds, $\max =511$ seconds for the mean and $\min =2.85 \%$, $\max$ $=4.61 \%$ for the standard deviation). It means that, because of the overestimation of the LWMs, many transport requests are waiting for a long time because areas are unable to get vehicles. Area 6, composed of furnaces which operate by batches of six lots, has the higher standard deviation (6.29\%). Once the processes are completed on a furnace, six vehicles are required to unload the machine and six vehicles to load the machine which explain the high variability. However, the standard deviations for Areas 2, 5 and 
Ben Chaabane, Dauzère-Pérès, Rullière, Lamiable, and Yugma

Table 2: Means and standard deviations of delivery times for different values of $L$

\begin{tabular}{|c|c|c|c|c|c|c|c|c|}
\cline { 2 - 9 } \multicolumn{1}{c|}{} & \multicolumn{9}{c|}{ Delivery Time } \\
\cline { 2 - 9 } \multicolumn{1}{c|}{} & \multicolumn{9}{|c|}{ Scenario 1 $(L=-1)$} & \multicolumn{2}{c|}{ Scenario 2 $(L=0)$} & \multicolumn{2}{c|}{ Scenario 3 $(L=0.2)$} & \multicolumn{2}{c|}{ Scenario 4 $(L=0.4)$} \\
\hline Area & Mean & Stand. Dev. & Mean & Stand. Dev. & Mean & Stand. Dev. & Mean & Stand. Dev. \\
\hline 1 & 389 & $4.36 \%$ & 354 & $2.61 \%$ & 257 & $1.62 \%$ & 299 & $2.88 \%$ \\
\hline 2 & $\mathbf{2 8 7}$ & $\mathbf{1 . 7 1 \%}$ & $\mathbf{3 3 0}$ & $\mathbf{1 . 8 4 \%}$ & $\mathbf{2 0 3}$ & $\mathbf{0 . 9 4 \%}$ & $\mathbf{3 0 1}$ & $\mathbf{2 . 1 3 \%}$ \\
\hline 3 & 447 & $4.61 \%$ & 432 & $2.86 \%$ & 301 & $1.43 \%$ & 378 & $3.03 \%$ \\
\hline 4 & 511 & $3.37 \%$ & 445 & $2.70 \%$ & 354 & $0.98 \%$ & 479 & $2.12 \%$ \\
\hline 5 & $\mathbf{3 4 9}$ & $\mathbf{2 . 7 7 \%}$ & $\mathbf{2 8 1}$ & $\mathbf{2 . 6 7 \%}$ & $\mathbf{1 4 1}$ & $\mathbf{1 . 3 9 \%}$ & $\mathbf{1 8 8}$ & $\mathbf{2 . 0 1 \%}$ \\
\hline 6 & $\mathbf{3 5 7}$ & $\mathbf{6 . 2 9 \%}$ & $\mathbf{3 0 7}$ & $\mathbf{1 . 9 3 \%}$ & $\mathbf{1 8 1}$ & $\mathbf{1 . 6 2 \%}$ & $\mathbf{4 5 0}$ & $\mathbf{3 . 9 5 \%}$ \\
\hline 7 & 390 & $4.38 \%$ & 245 & $3.03 \%$ & 180 & $2.63 \%$ & 267 & $2.48 \%$ \\
\hline 8 & 510 & $3.35 \%$ & 489 & $2.07 \%$ & 354 & $1.65 \%$ & 345 & $2.77 \%$ \\
\hline 9 & $\mathbf{5 7 6}$ & $\mathbf{1 . 7 3 \%}$ & $\mathbf{5 1 1}$ & $\mathbf{1 . 8 4 \%}$ & $\mathbf{1 2 7}$ & $\mathbf{1 . 5 8 \%}$ & $\mathbf{2 7 1}$ & $\mathbf{1 . 8 6 \%}$ \\
\hline 10 & 403 & $2.85 \%$ & 412 & $1.77 \%$ & 397 & $1.02 \%$ & 354 & $3.16 \%$ \\
\hline
\end{tabular}

9 are lower than for other areas $(\min =1.71 \%, \max =2.77 \%)$. These areas are specific since their high throughputs (processing times between 2 and 15 minutes) lead to a large number of transport requests and allow them to have relatively high LWMs to keep many vehicles at the expenses of other areas. The mean delivery time of Area 9 is relatively long (576 seconds) despite its high LWM (15 vehicles). Some analysis shows that this is because the high LWM leads to an accumulation of extra vehicles which causes a traffic jam.

In the second scenario, the degree of freedom $(L)$ is null, allowing more vehicles to be available to perform transport requests. Both means and standard deviations improved although the LWMs of areas with high throughputs have decreased (from 6 to 3 for area 2, from 8 to 4 for Area 5, from 7 to 4 for Area 6 and from 11 to 6 for Area 9). These changes allow other areas to keep vehicles and answer transport requests. However, this improvement is still insufficient because the variability is relatively high $(\mathrm{min}=$ $1.77 \%$, $\max =3.03 \%)$.

In the third scenario, the degree of freedom $(L)$ is equal to $20 \%$, i.e. only $80 \%$ of the transport fleet is used to ensure the stability of the system. The means and standard deviations of the delivery time are considerably improved $(\min =127$ seconds, $\max =397$ seconds for the mean and $\min =0.98 \%$, $\max =$ $2.63 \%$ for the standard deviation). This is due to the fact that vehicles do less balancing missions and transport resources are better allocated to satisfy transport requests.

In the last scenario, the degree of freedom $(L)$ is equal to $40 \%$. i.e. only $60 \%$ of the transport fleet is used to ensure the stability of the system. The results show a degradation in Area 2 (from 203 to 301 seconds for the mean and from $0.94 \%$ to $2.13 \%$ for the standard deviation), Area 5 (from 141 to 188 seconds for the mean and from $1.39 \%$ to $2.01 \%$ for the standard deviation), Area 9 (from 127 to 271 seconds for the mean and from $1.58 \%$ to $1.86 \%$ for the standard deviation) and Area 6 (from 181 to 450 seconds for the mean and from $1.62 \%$ to $3.95 \%$ for the standard deviation).

To conclude, selecting the right LMWs and an appropriate degree of freedom is critical for the performance of the Automated Material Handling System. Both the mean and the variability of the delivery times are influenced. In the next section, we study the impact of the different types of flows on the calculation of LWM.

\subsection{RELATIONSHIP BETWEEN LWM AND TRANSPORT TYPES}

In the previous section, scenarios do not take into account the interactions between areas. However, there are two types of transport requests: Some transport requests start and end in the same area, thus keeping the assigned vehicles available in the area, and the other transport requests start in one area and end in 
another area, thus reducing the number of vehicles in the origin area and increasing the number of vehicles in the destination area. Vehicles assigned to outside transport requests are lost for the origin area. The daily number of transport requests NbTrans $_{j}$ in area $j$ can be written:

$$
\text { NbTrans }_{j}=F I_{j}+F S_{j}
$$

where $F I_{j}$, respectively $F S_{i}$, is the number of transport requests staying in, respectively leaving, Area $j$. Moreover, let $F E_{j}$ be the number of transport requests from other areas entering Area $j$. The LWM should be impacted by $F S_{j}$ and $F E_{j}$ since, if there are more entering than leaving transport requests, i.e. $F S_{j}<F E_{j}$, than the reactivity of area $j$ to satisfy transport requests should be improved. On the opposite, i.e. $F S_{j}>F E_{j}$, than the reactivity of area $j$ to satisfy transport requests should be degraded. We take into account the different types of transport flows by changing (1) to:

$$
L W M_{j}=\frac{F I_{j}+\left(F S_{j}-F E_{j}\right)}{\sum_{i=1}^{n}\left(F I_{i}+\left(F S_{i}-F E_{i}\right)\right)} \times N T \times(1-L)
$$

It seems relevant to consider the dynamics of the system when calculating the LWMs to take the variability of transport requests into account. Hence, we also study the case where the LWMs are adjusted in real time in the simulation, depending on the current values of $F I_{j}, F S_{j}$ and $F E_{j}$.

Three scenarios are proposed which are detailed in Table 3:

- The first scenario is the third scenario of Section 4.2,

- The second scenario consists of calculating the LWMs using (2) only once,

- In the third scenario, LWMs are regularly adapted using (2) every 30 seconds.

Table 3: Three scenarios with different types of flows

\begin{tabular}{|c|c|c|c|c|c|c|}
\cline { 2 - 7 } \multicolumn{1}{c|}{} & \multicolumn{3}{c|}{ Flows } & \multicolumn{3}{c|}{ LWM } \\
\hline Area & FS & FI & FE & Scenario 1 & Scenario 2 & Scenario 3 \\
\hline 1 & 602 & 100 & 187 & 1 & 1 & $L W M_{1}(t)$ \\
\hline $\mathbf{2}$ & $\mathbf{8 4 6}$ & $\mathbf{4 9 9}$ & $\mathbf{8 4 0}$ & $\mathbf{2}$ & $\mathbf{1}$ & $L W M_{2}(t)$ \\
\hline 3 & 201 & 233 & 604 & 1 & 0 & $L W M_{3}(t)$ \\
\hline 4 & 800 & 157 & 1255 & 2 & 1 & $L W M_{4}(t)$ \\
\hline $\mathbf{5}$ & $\mathbf{1 4 9 9}$ & $\mathbf{4 0 4}$ & $\mathbf{8 9 7}$ & $\mathbf{3}$ & $\mathbf{2}$ & $L W M_{5}(t)$ \\
\hline $\mathbf{6}$ & $\mathbf{1 3 3 2}$ & $\mathbf{4 1 7}$ & $\mathbf{7 5 2}$ & $\mathbf{3}$ & $\mathbf{1}$ & $L W M_{6}(t)$ \\
\hline 7 & 929 & 624 & 1099 & 3 & 2 & $L W M_{7}(t)$ \\
\hline 8 & 133 & 297 & 352 & 1 & 1 & $L W M_{8}(t)$ \\
\hline $\mathbf{9}$ & $\mathbf{1 0 0 0}$ & $\mathbf{1 6 0 5}$ & $\mathbf{1 0 0 3}$ & $\mathbf{5}$ & $\mathbf{3}$ & $L W M_{9}(t)$ \\
\hline 10 & 101 & 300 & 716 & 1 & 0 & $L W M_{1} 0(t)$ \\
\hline
\end{tabular}

The number of transport requests matches the number of lot shipped per day in each area. The duration of the simulation is three days. Only the results of Areas 2, 5, 6 and 9 (data in bold in Table 3), characterized either by high throughput or the nature of the equipment(batch machines in Area 6), are presented in Table 4

Comparing the first two scenarios, note that the delivery time has not increased. In Areas 2 and 9, the number of transport requests leaving the area is nearly equal to the number of transport requests entering the area. Hence, even though the LWM is decreased (from 2 to 1 for Area 2, from 3 to 2 for Area 5, from 3 to 1 for Area 6 and from 5 to 3 for Area 9), the delivery time is not impacted. In the third scenario, the mean of the delivery time has considerably improved (minimum of 89 seconds and maximum of 151 seconds) with a reduced variability (minimum of $5.31 \%$ and maximum of $7.74 \%$ ). This illustrates that taking the dynamics of the system into account when calculating the LWMs is relevant. 
Ben Chaabane, Dauzère-Pérès, Rullière, Lamiable, and Yugma

Table 4: Simulation results for scenarios with different transport types

\begin{tabular}{|c|c|c|c|c|c|c|}
\cline { 2 - 7 } \multicolumn{1}{c|}{} & \multicolumn{4}{c|}{ Delivery Time } & \multicolumn{2}{c|}{ Scenario 3 } \\
\cline { 2 - 7 } \multicolumn{1}{c|}{ Scenario 1 } & \multicolumn{2}{c|}{ Scenario 2 } & \multicolumn{2}{c|}{ Scan } & Stand. Dev. \\
\hline Area & Mean & Stand. Dev. & Mean & Stand. Dev. & Mean & S.31\% \\
\hline 2 & 203 & $6.59 \%$ & 182 & $6.27 \%$ & 121 & $5.74 \%$ \\
\hline 5 & 141 & $9.72 \%$ & 136 & $10.68 \%$ & 111 & $7.16 \%$ \\
\hline 6 & 181 & $11.32 \%$ & 184 & $8.57 \%$ & 151 & $7.48 \%$ \\
\hline 9 & 127 & $11.06 \%$ & 113 & $8.12 \%$ & 89 & \\
\hline
\end{tabular}

\section{IMPACT OF THE HIGH WATER MARK}

The High Water Mark (HWM) is defined as the maximum number of available vehicles that can be in the area. This parameter is important to satisfy the transport requests in the area but also to satisfy transport requests in other areas. Moreover, the HWM helps to avoid area to be saturated with vehicles. The right values of the HWMs depend on the variability of the transport requests of the area. The HWM can be seen as a function of the LWM, the number of transport requests and the variability of the transport requests in each area. If $H W M_{i}>L W M_{i}$, then extra available vehicles are wanted in Area $i$. Thus, the setting of this parameter is important to cope with instant variability. We analyze two scenarios:

- The first scenario considers the impact of $H W M_{i}$ on the performance of Area $i$,

- The second scenario considers the impact of $H W M_{i}$ on the performance of its neighborhood.

The simulation is used to analyze the impact of the HWM on the delivery time. The reporting will be done for four specific areas: Areas 2, 5, 6 and 9. The number of replications is equal to 3. The duration of the simulation is one day.

Table 5: Simulation results for analyzing the HWM impact

\begin{tabular}{|c|c|c|c|c|c|c|c|c|}
\cline { 2 - 9 } \multicolumn{1}{c|}{} & \multicolumn{9}{c|}{ HWM(t) } \\
\cline { 2 - 9 } \multicolumn{1}{c|}{ LWM(t)+1 } & \multicolumn{2}{c|}{ LWM(t)+2 } & \multicolumn{2}{c|}{ LWM(t)+3 } & \multicolumn{2}{c|}{ LWM(t)+4 } \\
\cline { 2 - 9 } \multicolumn{1}{c|}{} & \multicolumn{2}{|c|}{ Delivery Time } & \multicolumn{2}{c|}{ Delivery Time } & \multicolumn{2}{c|}{ Delivery Time } & \multicolumn{2}{c|}{ Delivery Time } \\
\hline Area & Mean & Stand. Dev. & Mean & Stand. Dev. & Mean & Stand. Dev. & Mean & Stand. Dev. \\
\hline 2 & 118 & $8.29 \%$ & 107 & $4.27 \%$ & 106 & $4.35 \%$ & 108 & $4.76 \%$ \\
\hline 5 & 105 & $9.44 \%$ & 108 & $6.32 \%$ & 112 & $5.01 \%$ & 101 & $5.25 \%$ \\
\hline 6 & 138 & $8.70 \%$ & 130 & $6.98 \%$ & 121 & $8.13 \%$ & 111 & $6.81 \%$ \\
\hline 9 & 84 & $8.46 \%$ & 87 & $6.24 \%$ & 81 & $3.37 \%$ & 79 & $3.61 \%$ \\
\hline
\end{tabular}

From the results of Table 5, we note the improvement of the delivery time when the gap between HWM and LWM increases. It shows that the adjustment of the gap is very important to consider the variability of transport requests. In addition, note also that, when $H W M>L W M+3$, the delivery time starts to stagnate. When the HWM is larger than a given value, there is a risk of having too many vehicles without improving the delivery time.

Then, we study the impact of the HWM of a given area on its neighbor areas. The study is focused on Area 2, which is characterized by a large variability. In each simulation, we modify the HWMs of the three closest areas (Areas 3, 6 and 1). We consider the scenarios of Table 6. Table 7 shows the changes on the delivery times of Area 2. In the first scenario, the mean delivery time is 148 seconds, which decreases (121 seconds) when the HWM of Area 3 is increased in Scenario 2. The decrease of the mean delivery time (134 seconds) is not as large when the HWM of Area 6 is increased in Scenario 2, although Areas 3 and 6 are as close from Area 2. It can be explained by the fact that Area 3 has less transport requests 
than Area 6 and thus can provide more vehicles than Area 6. Area 1 being relatively far from Area 2, the mean delivery time in Area 2 does not really improve in Scenario 4 (144 seconds).

Table 6: Impact of varying HWM on its neighbor areas

\begin{tabular}{|c|c|c|c|}
\hline Scenario 1 & Scenario 2 & Scenario 3 & Scenario 4 \\
\hline $\begin{array}{c}H W M_{i}(t)=L W M_{i}(t), \\
i=(3,6,1)\end{array}$ & $H W M_{3}(t)=L W M_{3}(t)+3$ & $H W M_{6}(t)=L W M_{6}(t)+3$ & $H W M_{1}(t)=L W M_{1}(t)+3$ \\
\hline
\end{tabular}

Table 7: Scenarios to study the impact of HWM on its neighbor areas

\begin{tabular}{|c|c|c|c|c|c|c|c|c|}
\cline { 2 - 9 } \multicolumn{1}{c|}{} & \multicolumn{2}{c|}{ Scenario 1 } & \multicolumn{2}{c|}{ Scenario 2 } & \multicolumn{2}{c|}{ Scenario 3 } & \multicolumn{2}{c|}{ Scenario 4 } \\
\cline { 2 - 9 } \multicolumn{1}{c|}{ Delivery Time } & \multicolumn{2}{c|}{ Delivery Time } & \multicolumn{2}{c|}{ Delivery Time } & \multicolumn{2}{c|}{ Delivery Time } \\
\hline Area & Mean & Stand. Dev. & Mean & Stand. Dev. & Mean & Stand. Dev. & Mean & Stand. Dev. \\
\hline 2 & 148 & $27.98 \%$ & 121 & $20.22 \%$ & 134 & $21.61 \%$ & 144 & $30.19 \%$ \\
\hline
\end{tabular}

\section{CONCLUSION}

Using discrete event simulation, we showed the importance of carefully setting the key parameters of a minimum service transport policy in a unified AMHS of large semiconductor manufacturing facility. Both the average values and the variability of delivery times are strongly influenced. Formulas were proposed to calculate these parameters. More research is required to evaluate the relevance of these formulas. Future studies will also focus on formalizing the approach to provide a decision support tool that determines in real time when the parameters should be updated.

\section{Acknowledgments}

This study has been done within the framework of a joint collaboration of STMicroelectronics in Crolles, France, and the Center of Microelectronics in Provence of the Ecole des Mines de Saint-Etienne in Gardanne, France. The authors would like to thank the ANRT (Association Nationale de la Recherche et de la Technologie) which has partially financed this study.

\section{REFERENCES}

Hammel, C., T. Schmidt, and M. Schops. 2012. "Network Optimisation To Dynamic Simulation Of AMHS". In Proceedings of the 2012 Winter Simulation Conference, 4673-4781. Berlin (Germany).

Kahraman, A. F., A. Gosavi, and K. J. Oty. 2008. "Stochastic Modeling of An Automated Guided Vehicle System With One Vehicle And A Closed-Loop Path". Transactions On Automation Science And Engineering 5:504-518.

Kiba, J.-E., S. Dauzere-Peres, C. Yugma, and G. Lamiable. 2009. "Simulation Of Full 300mm Semiconductor Manufacturing Plant With Material Handling Constraints". In Proceedings of the 2009 Winter Simulation Conference, 1601-1609. Austin, Texas (USA).

Nazzal, D., and L. McGinnis. 2005. "Queuing Models Of Vehicles-Based Automated Material Handling Systems In Semiconductor Fabs". In Proceedings of the 2005 Winter Simulation Conference, 2464-2471. Orlando, Florida (USA).

Nazzal, D., and L. McGinnis. 2006. "An Analytical Model Of Vehicle-Based Automated Material Handling Systems In Semiconductor Fabs". In Proceedings of the 2006 Winter Simulation Conference, 1871-1879. Monterey, California (USA).

Nazzal, D., and L. McGinnis. 2007. "Expected Response Times For Closed-Loop Multivehicle AMHS". IEEE Transactions On Automation Science And Engineering 4:533-542. 
Wertz, R., C. Fischmann, F. Bottinger, and M. Karsperczyk. 2008. "Improved Empty Vehicle Balancing In Automated Material Handling Systems". In Proceedings of the 2008 International Conference on Computer Modeling and Simulation, 732-733. Cambridge (UK).

\section{AUTHOR BIOGRAPHIES}

AHMED BEN-CHAABANE is a Ph.D. student at Ecole Nationale des Mines de Saint-Etienne, France. $\mathrm{He}$ works as an Engineer in the Industrial Engineering Department at STMicroelectronics Crolles. He received the Industrial Engineering degree from Grenoble Institute of Technology,France, 2011. His Ph.D subject is about Automated Material Handling Systems modeling and optimization. His email address for these proceedings is ahmed.ben-chaabane@st.com.

STEPHANE DAUZERE-PERES is Professor and Director of the Center of Microelectronics in Provence of the EMSE. He received the Ph.D. degree from the Paul Sabatier University in Toulouse, France, in 1992; and the H.D.R. from the Pierre and Marie Curie University, Paris, France, in 1998. He was a Postdoctoral Fellow at the Massachusetts Institute of Technology, U.S.A., in 1992 and 1993, and Research Scientist at Erasmus University Rotterdam, The Netherlands, in 1994. He has been Associate Professor and Professor from 1994 to 2004 at the Ecole des Mines de Nantes in France where he headed the team Production and Logistic Systems (about 20 members) between 1999 and 2004 . He was invited Professor at the Norwegian School of Economics and Business Administration, Bergen, Norway, in 1999. Since March 2004, he is Professor at the Ecole des Mines de Saint-Etienne, where he headed the research department Manufacturing Sciences and Logistics (SFL, about 20 members) from 2004 to 2013. His research interests broadly include modeling and optimization of operations at various decision levels (from real-time to strategic) in manufacturing and logistics, with a special emphasis on semiconductor manufacturing. He has published 45 papers in international journals and contributed to more than 100 communications in conferences. Stéphane Dauzère-Pérès has coordinated multiple academic and industrial research projects, and also five conferences. His email address is Stephane.Dauzere-Peres@emse.fr.

CLAUDE YUGMA is an associate professor at the Provence Microelectronics Center of the École des Mines de Saint-Etienne. He received the Ph.D. degree from the Institut National Polytechnique of Grenoble, France, in 2003; He was a Postdoctoral student at the École Nationale Sup'erieure de G'enie Industriel, Grenoble, from 2003 to 2004 and from 2005 to 2006 at the Provence Microelectronics Center. His email address is Claude.Yugma@emse.fr.

LIONEL RULLIERE is an Automated Material Handling System team leader belonging to the Industrial Engineering Department of STMicroelectronics Crolles 300MM. During his studies, he works in 1996 on the evaluation and modeling of business strategies (simulations, cost model) in Mechanical Engineering department, Manufacturing design, Bath university, England. He received in 1997 a Manufacturing Systems Engineering degree from French Institute of Advanced Mechanical Engineering (IFMA)- Clermont-Ferrand, France. Between 1998 and 2000, he was an enterprise resource planning consultant in EDS- Lyon, France. He joined STMicroelectronics Crolles site in 2000 as a Line management Production Control, than a Shift Area Manufacturing Manager. Since 2004 he is working into Industrial Engineering Department. His email address for these proceedings is lionel.rulliere@st.com.

GILLES LAMIABLE is AMHS and Sorter Group Manager belonging to the Industrial Engineering Department of STMicroelectronics Crolles 300MM. He joined Crolles site in early 1992 as maintenance engineer. In 1997, he received is Engineering degree and introduced new operator interface concept in the 200mm line. Early 2001 as Methods manager, he participated to the AMHS supplier selection program for the new 300mm line and then was in charge of the specifications and installation in the Fab. In 2006, he 
Ben Chaabane, Dauzère-Pérès, Rullière, Lamiable, and Yugma

was in charge of the functional scenarios and operator interface of the system to assure the full integration into the manufacturing line.His email address for these proceedings is gilles.lamiable@st.com. 